\title{
Radicalisme religieux et pratiques d'écriture au début de l'époque moderne en France
}

\section{Xenia von Tippelskirch}

\section{(2) OpenEdition \\ 1 Journals}

Édition électronique

URL : http://journals.openedition.org/assr/22106

DOI : 10.4000/assr.22106

ISSN : $1777-5825$

Éditeur

Éditions de l'EHESS

\section{Édition imprimée}

Date de publication : 1 juin 2010

Pagination : 9-17

ISBN : 978-2-7132-2254-2

ISSN : 0335-5985

Référence électronique

Xenia von Tippelskirch, «Radicalisme religieux et pratiques d'écriture au début de l'époque moderne en France », Archives de sciences sociales des religions [En ligne], 150 | avril-juin 2010, mis en ligne le 14 septembre 2010, consulté le 20 avril 2019. URL : http://journals.openedition.org/assr/22106 ; DOI : 10.4000/assr.22106 


\section{Xenia von Tippelskirch}

\section{Radicalisme religieux et pratiques d'écriture au début de l'époque moderne en France}

Quand nous parlons aujourd'hui de "radicalisme » ou de "radicalités ", nous utilisons des termes qui ne sont pas du XVII ${ }^{\mathrm{e}}$ siècle. Selon les dictionnaires de l'âge classique, est " radical » ce " qui sert de base et de fondemens » (Furetière, 1968 : 1717), "qui a une vertu nutritive, ainsi qu'une racine " (Pomey, 1671 : 771) ; Richelet (1973 : 539) propose ainsi l'exemple suivant : "l'homme a radicalement la faculté de raisonner $\&$ de rire ». Les auteurs des dictionnaires se réfèrent donc en premier lieu à l'essence, à l'origine ; "radicalisme » comme manière de désigner une attitude politique n'étant attesté en France que depuis 1820, selon un usage dérivé de l'anglais où il apparaît vers la fin du XVIII ${ }^{\mathrm{e}}$ siècle ${ }^{1}$.

Si nous choisissons ici ce terme, en nous appuyant sur une autre définition proposée par Furetière : " radical » est également ce « qui vise à agir sur la cause profonde des effets qu'on veut modifier " (1968: 1717), ce qui est «drastique » et « absolu ", c'est pour ne pas juger a priori des actions des hommes et des femmes, issus du milieu catholique français, qui figurent au centre de nos études. En effet, les contemporains ne parlent pas de "radicaux », mais bien - quand ils les veulent critiquer - de "fanatiques », de personnes «extravagantes » 2 qui ont du mal à «tenir le juste milieu » (Boileau, 1737 : 178). Quand ils en parlent de manière positive, ils les décrivent comme des "saints".

Nous pouvons rappeler que, dans l'historiographie sur le XVII e siècle anglais, le terme de "radicalisme » fait depuis longtemps débat. Dans son introduction à La Révolution des saints (Walzer, 1987), le traducteur Vincent Giroud rappelle le sens politique que ce terme a en anglais. Il y explique pourquoi il a conservé l'usage des termes « radical » et « radicalisme » dans un contexte dix-septièmiste : "dans le langage anglo-américain (...) est un radical celui qui se reconnaît le droit de juger ce qui est vrai et mensonger, juste et injuste, légitime et illégitime, désirable et condamnable, sans soumission aucune aux conventions et aux autorités

1. On pourrait rappeler ici le travail fondamental de Halévy, 1995, sur le contexte anglais du XVIII ${ }^{\mathrm{e}}$ siècle.

2. Voir la contribution de L. Trigueros dans ce volume. 
établies, il ne sépare pas la défense des libertés individuelles de celle des libertés politiques et lie l'une et l'autre à la recherche du bien public et de la justice sociale. Associant la volonté de transformer les institutions, l'anti-autoritarisme, la critique du privilège, la méfiance à l'égard des défenseurs du pouvoir, l'attachement à la participation du peuple aux affaires de la nation, le radicalisme, quoiqu'il ait pu coïncider avec des projets révolutionnaires, relève d'une éthique du politique, plutôt qu'il ne désigne un mouvement régi par une fin déterminée » (id. : 13)

Les critiques soulevées par les travaux importants de E. P. Thompson et de Christopher Hill, résumées récemment par Glenn Burgess (2007), pointent plusieurs failles de la démarche passionnée des historiens marxistes britanniques ayant eu recours à ce terme de manière substantielle. Leur tentative pour retracer une véritable tradition de l'idéologie radicale du temps de la Révolution anglaise jusqu'à eux-mêmes, qui présume donc un passage des idées radicales d'une époque à l'autre, ne permettait pas de tenir compte des interactions probablement beaucoup plus significatives entre contemporains. L'assomption d'une identité de classe semble également problématique. Colin Davis (1982) tente de surpasser l'impasse en proposant une approche fonctionnaliste selon laquelle le radicalisme se définirait par : 1) une délégitimation de l'ordre sociopolitique ancien ; 2) la relégitimation d'un nouvel ordre sociopolitique ; et en proposant 3) un mécanisme de transfert de l'ordre ancien vers le nouveau. Il ne présuppose donc pas de lien effectif/ historique entre différents mouvements radicaux et ne les place pas dans une généalogie continue. En allant plus loin dans sa critique, Conal Condren (1994) dénonce l'anachronisme du concept. Selon lui, non seulement le terme radicalisme n'a pas été utilisé par les hommes de l'Ancien régime, mais le signifié moderne (désir d'innovation) ne correspondrait nullement à leurs attentes, qui visaient plutôt la conservation et la rénovation. On a aussi avancé que le fait d'avoir privilégié la dimension politique du radicalisme anglais a amené à négliger l'importance de la religion dans les conflits décrits (Burgess, 2007 : 11-12).

En reprenant pourtant le terme de radicalisme, pour aborder le XVII ${ }^{\mathrm{e}}$ siècle français, nous ne négligeons pas ces critiques : nous proposons d'aller au-delà de la contextualisation strictement intellectuelle qu'elles opposent à la démarche continuiste des historiens progressistes de la "révolution des saints ». L'anachronisme volontaire nous permet de suspendre le jugement, sans insinuer de généalogies idéologiques. Mais s'interroger sur le fonctionnement oriente l'attention vers les pratiques qui contribuaient alors à radicaliser l'expérience religieuse.

Nous voulons ainsi tenter ici une approche de plusieurs formes de radicalités religieuses sans prétendre qu'il s'agissait d'un mouvement commun. Au-delà des divisions entre courants religieux, il semble en effet que les pratiques se ressemblent ; autrement, et plus précisément dit, il existe des pratiques spécifiques qui ont les mêmes fonctions, agissent de manière semblable, sont adoptées de manière comparable, et suscitent des réactions analogues. Elles produisent des radicalités. 
L'abbé Jean-Jacques Boileau soutient, dans une lettre écrite entre la fin du $\mathrm{XVII}^{\mathrm{e}}$ et le tout début du XVIII ${ }^{\mathrm{e}}$ siècle, que «l'esprit humain tombe presque toujours dans les extrémitez. Il veut être ou dans une sécurité entière ou dans un doute général. Mais la raison \& la foi évitent également ces deux extrémités ; il y a des matières où l'on doit douter $\&$ craindre, parce qu'elles sont obscures, périlleuses, \& que nos dispositions nous sont inconnues; il y en a d'autres où il faut se tenir en repos, parce qu'elles ont été suffisamment éclaircies. » (1737 : 178). Il propose dès lors, comme moyen de trouver la paix de l'âme, d'exposer simplement tout ce qui procure du trouble à son confesseur et de suivre humblement les avis que ce dernier peut dispenser ${ }^{3}$. L'un des "problèmes » rencontrés par les acteurs sociaux, qui sont au centre de nos études, est bien le fait qu'ils n'ont pas toujours suivi l'avis d'un confesseur - ou bien que raison et foi n'étaient pas si nettement du même côté. Tous les hommes sont susceptibles de tomber dans le fanatisme, explique l'abbé François-Adrien Pluquet, quelques décennies plus tard : "les hérésies, les sectes, $\&$ les disputes de religion » alimentent le principe de fanatisme qui réside « au fond du cœur de tous les hommes » (1762:11).

Caractérisés par une grande détermination, ces radicaux semblent avoir conscience de faire partie d'une dynamique. Ils savent ce qu'ils veulent et pour quelles raisons ils le veulent, et leur désir de changement, c'est-à-dire d'une réforme radicale, se traduit fréquemment par une activité fébrile. Souvent contraints à la clandestinité, ils se réfugient dans l'anonymat de l'écrit, ou, au contraire, cherchent à obtenir un maximum de publicité grâce à la publication de leurs pensées. De là naissent des polygraphes, accusés par leurs adversaires de " parler trop d'euxmêmes " ${ }^{4}$. Leur activité les porte à pratiquer intensivement l'écriture, aussi bien que la réécriture. En même temps, les radicaux deviennent des objets de discussion, leurs écrits suscitent des réactions écrites, ils entrent dans des débats : c'est aussi dans cette perspective qu'il semble intéressant d'analyser la radicalité et le caractère subversif de certains textes, face à la radicalisation des différents pouvoirs.

On peut citer à ce sujet un homme particulièrement conscient de cette importance spécifique de l'écriture, dont il était lui-même un praticien chevronné. Il s'agit de Pierre Poiret, ancien pasteur protestant converti à un mysticisme plutôt catholique, même s'il a continué toute sa vie à bouger en marge de ce qui était considéré comme catholique par les institutions catholiques elles-mêmes. Il s'était chargé de l'édition d'une série de textes mystiques, des œuvres complètes d'Antoinette Bourignon et de Jeanne-Marie Guyon, dont il publie, en 1720, la vie $^{5}$. Dans sa préface à cette vie de Mme Guyon, Poiret décrit de manière très

3. Sur Boileau, voir Tamizey de Larroque, 1877 ; Durengues, 1907.

4. Cette accusation est évoquée par Pierre Poiret dans son apologie d'Antoinette Bourignon, auteure particulièrement prolifique (Bourignon, 1683, 49).

5. Cf. sur Poiret, Chevallier, 2000 ; sur la Vie et ses publications, voir les notes introductives de Dominique Tronc dans Guyon, 2001. 
lucide l'un des effets de la publication massive des querelles religieuses. Son point de vue est celui d'une personne fortement engagée dans le débat écrit, en marge de ce qu'on pourrait appeler un catholicisme modéré, qui croit toujours à sa mission - et qui regarde avec une certaine distance (après une vingtaine d'années) des dynamiques dont il a été partie prenante à plein titre :

«(...) on a cru durant un assez longtems avoir éteint $\&$ suprimé entierement ce qui regardoit les écrits $\&$ les faits de Madame Guion, a quoi on ne pensoit plus que par hazard comme à une fable passée, ou à une espece de comédie pleine d'extravagances qui s'étoit jouée vers la fin du dernier siècle \& terminée assez tragiquement. Mais Dieu, qui ne vouloit point que les grandes $\&$ salutaires vérités, qu'il avoit dessein de communiquer aux ames de bonne volonté par cet insigne organe de son Esprit Saint, demeurassent stériles, s'est servi pour les faire revivre \& pour en répandre par tout la connoissance, des mêmes personnes qui avoient cru les éteindre, \& des mêmes moiens dont ils s'étoient servi pour cet éfet. Car outre quelques uns de ses papiers qu'ils communiquèrent à des particuliers, les ouvrages qu'ils publièrent eux-mêmes à l'encontre aiant passé dans les pais étrangers, portèrent la curiosité de plusieurs, mêmes entre des personnes de considération à vouloir un peu pénétrer le fond d'une affaire qui avoit fait depuis peu un si grand bruit. Ce qui les aiant engagé à rechercher ses écrits, \& à lire sans préjugé ceux qu'ils purent trouver, ils en furent tellement touchés, qu'ils firent leurs éforts pour en découvrir $\&$ ensuite rendre publics pour la gloire de Dieu $\&$ le bien éternel de ceux qui veulent faire leur salut, tout autant qu'ils pourroient en recouvrer." (Poiret, $1720: \mathrm{XV}$ ).

Parlant d'écriture, Poiret parle à la fois de "personnes » et de "moyens » qui ont agi en quelque sorte au-delà d'eux-mêmes. Les détracteurs mêmes de Mme Guyon ont servi sa cause, puisque, en publiant des textes contre elle, ils ont alimenté la curiosité et finalement la croyance, la " fable » ou la "comédie » devenant une "vérité ", que d'autres acteurs devaient se donner la charge de rendre publique. La question qui se posait pour les "croyants » était celle du discernement entre la vérité et ce qui ne fait que lui ressembler. Une fois discernée, cette vérité absolue était à défendre par tous les moyens, en accumulant des preuves pour convertir ceux qui demeuraient dans une sorte d'obscurité - cette perspective affleure encore chez Henri Bremond dans l'Histoire littéraire du sentiment religieux (2006), qui a le mérite d'avoir attiré l'attention sur la production des mystiques du XVII ${ }^{\mathrm{e}}$ siècle, mais dont l'interprétation est fortement infléchie par le projet, pris entre positions intégralistes et centristes du début du XX $\mathrm{XX}^{\mathrm{e}}$ siècle, de retrouver l'histoire du "vrai » mysticisme (Ribard, 2002 ; Houdard, 2008 : 15-16). Dans le but d'affirmer la vérité, les radicaux, ou les croyants radicalisés, agissaient avec des méthodes bien « littéraires », et se trouvaient ainsi confrontés au fait que les mots peuvent être détournés, que les textes ont une matérialité spécifique, que la «fiction » pouvait jouer pour... ou contre eux !

Une série de questions découlent de la prise en compte sérieuse du lien entre pratiques religieuses d'écriture et positions radicales. Quel rôle attribuait-on, à l'époque, à l'écriture pour définir les limites entre pratiques orthodoxes et hétérodoxes ? Comment manipulait-on les écrits pour se distinguer dans le champ religieux ? Comment les dynamiques dissidentes s'inscrivent-elles dans l'écriture? 
Y a-t-il des fonctions spécifiques de l'écrit dans le champ religieux ? Y a-t-il des écrits qui peuvent fonctionner comme indices de radicalité ? On peut également se demander quel rôle est donné à l'inspiration divine dans l'action de ces écrits : elle peut servir de justification, de prétexte ou de fondement épistémologique, comme par exemple dans le cas de Pierre Poiret qui défend l'idée que l'érudition ne peut réussir à établir toute la vérité s'il n'y a pas, aussi, intervention divine. On peut, enfin, s'efforcer de proposer des analyses qui tiennent compte de ces différentes lectures possibles : peut-on caractériser le radicalisme comme " désordre » par rapport à un " ordre " social impliquant aussi des règles d'écriture ?

En nous fondant sur des études de cas précises, notre équipe interdisciplinaire (historiens de l'écrit aussi bien qu'historiens du fait religieux, littéraires, spécialistes de la théologie de l'époque moderne) souhaite nuancer un certain nombre de visions simplificatrices et aller au-delà du constat purement quantitatif de l'abondance des écrits liés au radicalisme religieux. Nous voudrions pour cela mettre à profit l'effet de distance, l'aliénation provoquée par ces écrits auxquels il est difficile d'adhérer, et qui ne permettent pas d'identification immédiate avec l'auteur, le scripteur ou le narrateur, pour examiner le lien entre radicalisation et acte d'écriture et le rôle de l'écrit dans la définition des limites du radicalisme. Il nous semble possible de montrer que pratiques et croyances religieuses sont rendues radicales par des pratiques d'écriture particulières. L'écriture, ici, crée une dissidence, avant même qu'une dissidence s'organise dans une écriture.

Deux questions, deux types d'hypothèse découlent de cette inversion heuristique de la vision commune. Écrire d'une manière spécifique serait-ce donc, pour le dire de manière abrégée et provocatrice, être radical ? Ou encore : l'écriture peut-elle radicaliser, transformer des pratiquants/croyants, quelle que soit leur croyance, en radicaux ? Ces questions nous permettent d'esquisser une nouvelle approche des dissidences religieuses, en faisant émerger des dynamiques insues.

La première nous fait suivre les traces d'écrivains ou de personnages qui se présentent comme tels, notamment François Davant dont s'occupent Lionel Trigueros et Bérengère Parmentier dans ce numéro thématique. Dans son étude, L. Trigueros montre que l'analyse policière des écrits de Davant ne désigne pas seulement la critique violente de l'ordre politique et social qui y est contenue, mais qu'elle identifie bel et bien la pratique de la réécriture adoptée par Davant comme activité de subversion : en proposant des réécritures radicales, Davant critique les pouvoirs en place tout en mettant en cause la pratique des textes de son époque. Bérengère Parmentier lit l'affaire de Davant à la lumière du cas de deux autres scribes, Simon Morin et François Doche : ces trois individus franchissent les limites que leur assigne leur statut social en essayant de se construire une nouvelle identité par l'écriture. Il était impossible pour ces hommes "sans études " de se concevoir comme des académiciens, mais ils pouvaient bien se concevoir comme des prophètes. Leur écriture religieuse est efficace dans l'activisme de proximité, mais ils ne possèdent pas les codes de la pratique de l'écriture comme pratique 
sociale; à l'exception du contexte de la Fronde, qui leur offre un espace de réception favorable, leurs textes restent illisibles. Selon B. Parmentier, la radicalité de ces personnages réside dans le fait de s'attribuer une autorité, grâce à un pouvoir pourtant dominé, celui de l'écriture, avant d'avoir conquis un statut.

Les considérations de Jean-Pascal Gay partent du fait que les transformations des rapports aux autorités et des configurations sociopolitiques des discours et des savoirs, à la fin du XVII ${ }^{\mathrm{e}}$ siècle, ont brouillé le statut social et ecclésial des théologiens. L'originalité des pratiques d'écriture des religieux radicaux - qui revendiquent une application stricte des principes théologiques et qui se prononcent contre l'indulgence morale -, se révèle aussi dans leurs choix textuels : ils n'exposent pas leur radicalité dans des sommes, mais dans des traités sur une question particulière qui les positionnent dans le jeu politique des controverses. J.-P. Gay souligne qu'au début du XVIII ${ }^{\mathrm{e}}$ siècle l'autorité des théologiens est régulièrement invoquée grâce et non pas malgré leur implication dans des polémiques : cette implication devient discours religieux légitime.

La seconde question nous permet de considérer l'impact des témoignages et des historiographies sur les occasions où l'écriture, voire une simple signature dans le cas analysé par Alain Cantillon, pose des limites. A. Cantillon s'intéresse dans sa contribution à la mobilisation de l'écrit dans le conflit de radicalités provoqué par le fameux Formulaire (sur les cinq propositions contenues dans le livre de Jansénius). D'un côté, les institutions ecclésiastiques essayent en quelque sorte de faire précipiter la croyance dans l'écriture, en demandant avec la souscription une forme uniforme (et visible) de la profession de foi. De l'autre, les lettres attribuées à la sœur Jacqueline Pascal, précieusement conservées et transmises par Port-Royal, présentent le problème de la souscription du formulaire et la tentative d'inventer une solution à Port-Royal : la revendication de la radicalité individuelle d'une conscience trouve son expression dans des lettres spirituelles où la «corresponsabilité » est essentielle.

Nous considérons ici des pratiques d'écriture (dans le sens le plus restreint) ${ }^{6}$, mais aussi des pratiques de publication (dans le sens de Jouhaud/Viala, 2002), de circulation de textes et de savoirs. L'étude de la publication du récit de conversion du curé Jean-Ignace de Jougla (Xenia von Tippelskirch) suggère que c'est l'action d'écriture et de publication de cette conversion qui radicalise l'expérience et qui délimite des camps sociaux. La description de la conversion acquiert ici la valeur d'un factum pouvant être mobilisé à l'intérieur d'une controverse qui vise à redéfinir les limites de l'orthodoxie. C'est grâce à l'étude d'approbations, d'épitres dédicatoires, de privilèges et de choix typographiques que Dinah Ribard peut montrer qu'il faut, dès lors qu'il est question de radicalité, aller au-delà d'une analyse du fonctionnement des institutions et se concentrer sur les pratiques et leurs effets

6. Michel de Certeau a considéré les difficultés découlant du fait de vouloir rendre compte par écrit de l'expérience indicible de l'union avec Dieu (1982). 
propres. La réalité de l'imprimé (sa matérialité et sa dimension policière) fait partie de l'action de l'écriture spirituelle de la seconde moitié du XVII siècle. Ainsi, des écrits, qui ne sont pas garantis par des institutions ou des communautés reconnues, peuvent-ils être autorisés grâce à leur mise en circulation et à leur réception possible par des lecteurs amateurs. La supercherie littéraire permet de contourner les voies contrôlées de la controverse et montre aussi une compétence sociale importante des radicaux.

L'écriture, pratique importante pour montrer son appartenance " au monde » à la fin $\mathrm{du} \mathrm{XVII}^{\mathrm{e}}$ siècle, se révèle ainsi également pratique étroitement liée à l'histoire sociale religieuse.

\author{
Xenia von TIPPELSKIRCH \\ Rubruniversität Bochum \\ xenia.vontippelskirch@rub.de
}

\title{
Bibliographie
}

Sources :

[Boileau Jean-Jacques], 1737, Lettres de Monsieur B. sur differens sujets de morale et de pieté, Paris, Charles Osmont.

BouRIGNON Antoinette, 1683, La vie de Damlle Antoinette Bourignon. Ecrite partie par elle-meme, partie par une personne de sa connoissance [...], Amsterdam, Jean Riewerts, Pierre Arents.

FURETIÈRE Antoine, 1968, Essais d'un dictionnaire universel [1687], Genève, Slatkine Reprints.

GuYON Jeanne-Marie, 2001, La Vie par elle-même (éd. critique par D. Tronc), Paris, Honoré Champion.

Pluquet F. A. A. l'abbé, 1762, Memoire pour servir à l'histoire des égarements de l'esprit humain par rapport a la religion chrétienne: ou dictionnaire des hérésies, des erreurs et des schismes; précédé d'un discours dans lequel on recherche quelle a été la religion primitive des hommes, les changemens qu'elle a soufferts jusqu'à la naissance du christianisme; les causes générales, les liaisons \& les effets des hérésies qui ont divisé les chrétiens, Paris, Chez Nyon à l'occasion, chez Barrois a la Ville de Nevers, P. Fr. Didot le jeune à saint Augustin.

POMEY François-Antoine, 1671, Le Dictionnaire royal augmenté, seconde édition, enrichie d'un grand nombre d'expressions élégantes, de quantité de mots françois nouvellement introduits, des termes des arts et de cinquante descriptions, Lyon, A. Molin.

[Poiret Pierre], 1720, Préface à La Vie de Madame J. M. B. de la Mothe Guion. Écrite par Elle-même, Cologne, Jean de la Pierre.

Poiret Pierre, [1700] 2005, Écrits sur la Théologie mystique. Préface, Lettre, Catalogue, introduction et notes par Marjolaine Chevallier, Grenoble, Éditions Jérôme Millon.

RichelEt Pierre, [1732] 1973, Dictionnaire de la langue françoise ancienne et moderne (reprod. de l'édition d'Amsterdam : aux dépens de la Compagnie), Paris, FranceExpansion. 
Études :

BREmond Henri, 2006, Histoire littéraire du sentiment religieux en France, Grenoble, Éditions Jérôme Millon.

Burgess Glenn, 2007, «Introduction » in Burgess G., Festenstein M. (éds.), English Radicalism, 1550-1850, Cambridge, Cambridge University Press, pp. 1-16.

Certeau Michel de, 1982, La Fable mystique XVI ${ }^{e}$-XVII ${ }^{e}$ siècle, Paris, Éditions Gallimard.

Chevallier Marjolaine, 2000, "Pierre Poiret (1646-1719)", Revue d'histoire et de philosophie religieuses, 80-4, pp. 503-525.

Condren Conal, 1994, The Language of Politics in Seventeenth Century England, Basingstoke-London, Macmillan Press.

DAvIs James Colin, 1982, "Radicalism in a Traditional Society: The Evaluation of Radical Thought in the English Commonwealth 1649-1660 ", History of Political Thought, 3, pp. 193-213.

Durengues Antoine, 1907, Monsieur Boileau de l'archevêché, Agen, Imprimerie moderne.

Halévy Elie, 1995, La formation du radicalisme philosophique (1901), Paris, Presses Universitaires de France, coll. "Philosophie morale».

Jouhaud Christian, Viala Alain, (dirs.), 2002, De la publication entre Renaissance et Lumières, Paris, Éditions Fayard.

RIBARD Dinah, 2002, "L'anachronique ou l'éternel. L'abbé Bremond et l'histoire littéraire », Cahiers du Centre de Recherches Historiques, 28-29, Quelques "dix-septième siècle »: fabrications, usages et réemplois, pp. 39-54.

Tamizey de Larroque Philippe, 1877, Notes sur la vie et les ouvrages de l'abbé JeanJacques Boileau publiées avec divers documents inédits, Paris, Aubry.

WaLzer Michael, 1987, La Révolution des saints. Éthique protestante et radicalisme politique, Paris, Belin. [The Revolution of the Saints: a Study in the Origins of Radical Politics, 1965].

\section{Résumé}

Entreprendre de réformer la religion et ses pratiques de manière radicale entraîne des conflits avec l'Église; ces conflits, au XVII siècle, sont souvent menés sur le terrain de l'écrit, et l'écrit en est un des enjeux. Penser les dissidences religieuses de l'époque moderne sur ce terrain, en termes de "radicalisme religieux " et de "radicalité ", permet de s'interroger sur des fonctionnements: les études ici réunies se concentrent sur les pratiques qui contribuaient alors à radicaliser l'expérience religieuse. De là toute une série de questions. Quel rôle attribuait-on à l'époque à l'écriture pour définir les limites entre pratiques orthodoxes et pratiques hétérodoxes? Comment les dynamiques dissidentes s'inscrivent-elles dans l'écriture? Des écrits peuvent-ils fonctionner comme indices de radicalité ? L'écriture peut-elle transformer des croyants, quelle que soit leur croyance, en radicaux? Il s'agit ainsi d'analyser les places que peuvent prendre des acteurs sociaux historiquement situés sur un théâtre constitué par la mise en action d'écrits différents.

Mots-clés : radicalisme religieux, pratiques d'écriture, radicalité, dissidence religieuse, hétérodoxie. 


\section{Abstract}

The attempt to radically reform religion and its practices entails conflict with the established Churches; in $17^{\text {th }}$-century France these conflicts were often carried out in written texts, texts themselves also being contested matters. Thinking about religious dissidence in terms of "religious radicalism" and "radicality" allows us to investigate its modes of operation. The studies brought together here focus on the practices that contributed to the radicalization of religious experiences, leading to several questions: What role did writing in Early Modern France play in defining the boundaries between orthodox and heterodox practices? How did dissident practices inscribe themselves into texts? Can texts be treated as markers of radicalism? Can writing transform believers, whatever they may believe in, into radicals? The essays that follow analyze the positions that historical protagonists could occupy in the theatre constituted by different writing actions.

Key words: religious radicalism, writing practices, religious dissent, heterodoxy.

\section{Resumen}

Emprender una reforma de la religión y sus prácticas de manera radical implica conflictos con la Iglesia; estos conflictos, en el siglo XVII, son a menudo llevados al terreno de lo escrito, y lo escrito se vuelve una de sus apuestas. Pensar las disidencias religiosas de la época moderna en este terreno, en términos de "radicalismo religioso" $y$ de "radicalidad" permite preguntarse sobre ciertos funcionamientos: los estudios aquí reunidos se concentran en las prácticas que contribuían entonces a radicalizar la experiencia religiosa. De abi toda una serie de interrogantes. ¿Qué rol se atribuía en la época a la escritura para definir los limites entre prácticas ortodoxas y prácticas heterodoxas? ¿Cómo se inscriben en la escritura las dinámicas disidentes? ¿Pueden funcionar los escritos como índice de radicalidad? ¿Puede la escritura transformar a los creyentes, sea cual sea su creencia, en radicales? Se trata asi de analizar los lugares que pueden tomar actores sociales históricamente situados en un teatro constituido por la puesta en acción de escritos diferentes.

Palabras clave: radicalismo religioso, prácticas de escritura, radicalidad, disidencia religiosa, heterodoxia. 